\title{
Chaos, thermodynamics and quantum mechanics: an application to celestial dynamics
}

\author{
David Vitali ${ }^{1}$ and Paolo Grigolini ${ }^{2,3,4}$ \\ * Dipartimento di Matematica e Fisica, Università di Camerino, via Madonna delle Carceri I-62032 Camerino \\ and Istituto Nazionale di Fisica della Materia, Camerino, Italy \\ ${ }^{1}$ Dipartimento di Fisica, Università di Pisa, Piazza Torricelli 2, 56126 Pisa, Italy \\ 2 Istituto di Biofisica del Consiglio Nazionale delle Ricerche, via San Lorenzo 28, 56127 Pisa, Italy \\ ${ }^{3}$ Department of Physics, University of North Texas, P.O. Box 5638, Denton, Texas 76203
}

We address the issue of the quantum-classical correspondence in chaotic systems using, as recently done by Zurek [e-print quant-ph/9802054, the solar system as a whole as a case study: this author shows that the classicality of the planetary motion is ensured by the environment-induced decoherence. We show that equivalent results are provided by the theories of spontaneous fluctuations and that these latter theories, in some cases, result in a still faster process of decoherence. We show that, as an additional benefit, the assumption of spontaneous fluctuation makes it possible to genuinely derive thermodynamics from mechanics, namely, without implicitly assuming thermodynamics.

\section{INTRODUCTION}

The problem of how the classical macroscopic world emerges from the quantum substrate is the main problem of the interpretation of quantum mechanics and it is still the subject of an intense debate [1]. A well known conceptual difficulty stems from the quantum states which are linear superpositions of macroscopically distinguishable properties. Although these states have never been observed, they are, according to quantum mechanics, a legitimate physical condition, thereby resulting in the well known paradox of the Schrödinger cat [2] and, more in general, of the measurement processes [3].

Another controversial, and more subtle, problem is as to whether or not the correspondence principle, namely, the alleged physical property that makes the quantum mechanical predictions on macroscopic systems identical to those of classical mechanics, applies to the crucial case of classically chaotic systems. There exists a general agreement on the following fact: a quantum system with a classical-like initial condition, i.e. a wave packet localized and smooth over scales much larger than $\hbar$, will depart, sooner or later, from this classical condition as a natural effect of time evolution. The object of debate is given by the estimate of this time. According to some authors (see [4] and references therein), relevant discrepancies between classical and quantum motion occur only after a "relaxation time" $t_{R}$ proportional to some (system-dependent) power of the ratio $A / \hbar$, where $A$ is some characteristic action. For macroscopic systems this ratio is always so large as to make the correspon- dence principle essentially safe. According to Zurek and Paz [5,6] and Zurek [7] instead, the quantum systems that would be classically chaotic, are expected to depart from the classical-like initial condition at a time depending logarithmically on the ratio $A / \hbar, t_{Q} \simeq \lambda^{-1} \ln (A / \hbar)$ ( $\lambda$ is the relevant Lyapunov exponent) and consequently much sooner. This logarithmic time is denoted by the subscript $Q$ to stress its meaning of transition from classical to quantum mechanics. This time can be uncomfortably short even for macroscopic systems, so short as to result in a new kind of quantum paradox [8]: deterministic chaos would establish quantum correlations among macroscopically distinct regions of the classical phase space of these systems.

This new paradox would break the correspondence principle and Zurek and Paz [5]6] and Zurek [7] state that in this case an efficient way of settling the paradox is offered by the theory of environmental decoherence [1],9]. The tenet of this theory is based on the observation that it is impossible to isolate completely a macroscopic system from the environment and that this unescapable interaction results in fluctuations, which, in turn, result in the rapid decay of the quantum coherence among macroscopically distinct components of the same quantum state. Decoherence quickly transforms a linear superposition of macroscopically distinguishable properties, for instance positions, into the corresponding classical statistical mixture; it represents therefore the practical solution of the Schrödinger cat paradox, even if from a conceptual and interpretational point of view, many problems are still open (see for example [10,11).

Zurek and Paz support this view with suggestive examples borrowed from celestial dynamics [6,7]. There is now in fact well-established numerical evidence of chaotic motion of celestial bodies, such as the chaotic tumbling of Hyperion, one of the moons of Saturn 12 , the orbital motion of Chiron in the asteroid belt 13. and the dynamics of the solar system as a whole 114,15. Quantum mechanics is judged to be more general than classical physics; furthermore the elementary constituents of these systems must obey quantum laws. Thus, we are forced to consider these systems as being quantum, and we are led to estimate the corresponding logarithmic time. The surprising conclusion is that this time turns out to be much shorter than the age of the systems themselves, since $t_{Q} \simeq 7 \cdot 10^{8}$ years for the solar system and $t_{Q} \simeq 20$ years for Hyperion.

According to quantum mechanics, as a consequence 
of this fact, the present state of Hyperion should be a coherent superposition of macroscopically distinct orientations of its major axis and, much more paradoxically, in the case of the planets of the solar system the wave function should have spread all over their orbits! The solution of the paradox given by Zurek and Paz in [6,7] is based on decoherence: even the tiny interaction with the interplanetary gas is sufficient to achieve the destruction of quantum coherence over macroscopic distances so as to obtain a localized wavepacket moving according to Newton laws. This takes place at a time much smaller than the logarithmic time $t_{Q}$ and any transition from classical to quantum mechanics is made impossible afterward.

Several aspects of the proposal by Zurek and Paz to make the quantum-classical correspondence safe by means of the environmental fluctuations have been recently criticized [4, 16]. For this reason, in this letter we plan to derive first of all the same physical conclusions from an approach bypassing, in part, these criticisms. We shall show that the time $t_{Q}$ is the correct estimate for the correspondence breakdown time if the condition of strong chaos case is ensured (i.e. when the classical phase space is filled by the chaotic sea with no appreciable stable island) and we shall consequently provide new arguments in favor of fluctuations as a proper route to the correspondence principle. However, and this is an other element of novelty of this letter, we shall consider also the possibility that these essential fluctuations might have a non-environmental origin. This means that we shall consider some models of spontaneous wavefunction reduction 17 20]. These theories generalize quantum mechanics by adding stochastic and nonlinear terms to the Schrödinger equation. We shall show that all these models make the solar system obey the quantumclassical correspondence, in the same way as the theory of environment-induced decoherence does. In particular the gravitation-induced collapse theory, a model originally introduced by Diósi and Penrose [19], and later improved by Ghirardi, Grassi and Rimini [20], turns out to be more efficient than the environmental process discussed by Zurek in [7, yielding in fact a much faster transition from the quantum to the classical domain. This is of special interest because this is the first dynamical system where the decoherence induced by spontaneous fluctuations is shown to become more important than the environmental decoherence 11, 21, 23.

To properly point out the significance of this aspect, it is worth mentioning one of the most frequent criticisms of the theories of spontaneous wave-functions collapses. All these generalizations of quantum mechanics have been developed by assigning ad hoc values to the parameters establishing the strength of the corrections to ordinary quantum mechanics. These choices serve the important purpose of making these theories pass all possible experimental tests (namely to coincide with quantum mechanics where this theory agrees with experimental results). As a consequence, the intensity of the corrections is so weak as to make it difficult to prove them with experi- ments. Furthermore, their key action is usually blurred by environmental decoherence, which is expected to be always predominant 21 23. We show that in the case of solar system it is not so.

Of course, this conclusion does not rule out the theory of environmental decoherence, since it is not possible to design an experiment aiming at assessing whether the solar system is made classical by environmental or spontaneous fluctuations. As argued in Section IV, we think that the choice of the theory of spontaneous fluctuations can be done only on the basis of theoretical arguments concerning the problem of unification of thermodynamics and mechanics as well as that of classical and quantum mechanics.

The structure of this letter is as follows. In Section II we briefly review the original work of Zurek and Paz [5] and some of the criticisms raised by this work [4, 16. Section III illustrates more general heuristic arguments, which yield the same conclusions as those by Zurek and Paz in the case of strong chaos. In this Section we also point out the essential role of coarse-graining processes for the foundation of thermodynamics. Section IV shows that the theory of spontaneous fluctuations leads to results formally equivalent to those of Section II, and that in the case of solar system, spontaneous fluctuations are more efficient than environmental fluctuations to ensure the quantum-classical correspondence. Finally, Section V summarizes the main results of this letter and discusses their possible consequences.

\section{TIME EVOLUTION OF THE WIGNER QUASIPROBABILITY AND LOGARITHMIC TIME}

Let us briefly review the arguments used by Zurek and Paz [5 [7] to derive the logarithmic time, and those used by them to settle the ensuing paradox as well. It is convenient to adopt the Wigner formalism and write the time evolution of the quantum system in terms of the corresponding quasi-probability $W(x, p)$. Furthermore, let us consider for simplicity the one-dimensional motion of a body under the influence of the analytical potential $V(x)$ and of a coupling, through the coordinate $x$, with a thermal environment. The resulting evolution equation is 24.

$$
\begin{aligned}
& \dot{W}=\{H, W\}_{P B}+\sum_{n \geq 1}^{\infty} \frac{\left(-\hbar^{2}\right)^{n}}{2^{2 n}(2 n+1) !}\left(\frac{\partial^{2 n+1} V}{\partial x^{2 n+1}}\right) \frac{\partial^{2 n+1} W}{\partial p^{2 n+1}} \\
& +2 \gamma \frac{\partial}{\partial p}(p W)+D \frac{\partial^{2} W}{\partial p^{2}}
\end{aligned}
$$

where $\{H, W\}_{P B}$ denotes the classical Poisson brackets, the second term is the non-classical, or Moyal term, and the last two terms describe the effects of the environment, producing (respectively) dissipation and diffusion. 
The analysis of Zurek and Paz concerns the so-called "reversible classical limit", in which the relaxation rate $\gamma$ tends to zero, with the diffusion coefficient $D=2 M \gamma K T$ kept constant. Furthermore, they set $t \ll 1 / \gamma$, so as to neglect the relaxation term of Eq. (11).

Zurek and Paz set for the system an initial condition compatible with the assumption that this is a classical "macroscopic" state. This means a Gaussian wave packet that in the corresponding classical phase space happens to be round and smooth over scales much larger than $\hbar$. Notice that the momentum and the coordinate widths, denoted by $\sigma_{p}(0)$ and $\sigma_{x}(0)$, respectively, are such that $\sigma_{x}(0) \sigma_{p}(0) \gg \hbar$. Even if we set $D=0$, the initial time evolution of the system is indistinguishable from that predicted by classical physics. This is so because the second term on the r.h.s. of Eq. (1), the Moyal term, involves derivatives of a smooth function and $\hbar$ is negligible compared to the classical action, thereby resulting in extremely small corrections to classical physics. However, as an effect of the fragmentation process resulting from the chaotic nature of the classical dynamics 25], the Moyal term becomes increasingly larger upon increase of time. In fact classical evolution implies that the exponential contraction along a given phase-space direction is balanced by an exponential dilatation along another directions so as to fulfill the classical prescription that the phase-space volume has to be conserved. Let us assume, for instance, the momentum squeezing $\Delta p(t)=\sigma_{p}(0) \exp (-\lambda t)$, where $\lambda$ is the relevant Lyapunov exponent. As a consequence, the quasi-probability $W(x, p)$ dilates in the $x$ direction so as to extend the quantum coherence to the distance $l(t) \simeq \hbar / \Delta p(t)$. When this coherence length $l(t)$ becomes of the order of the nonlinearity parameter $\chi$ defined by

$$
\chi \simeq \sqrt{\frac{\partial V / \partial x}{\partial^{3} V / \partial x^{3}}},
$$

the Moyal term is no more negligible and quantum dynamics begin departing from classical physics. Zurek and Paz show that this condition is realized at the logarithmic time $t_{Q}$, whose explicit expression is

$$
t_{Q}=\frac{1}{\lambda} \ln \left(\frac{\chi \sigma_{p}(0)}{\hbar}\right) .
$$

As pointed out in Section I, this is a state of affair dangerous for the quantum-classical correspondence. Thus, Zurek and Paz set $D>0$. Under this new condition, the classical fragmentation cannot proceed indefinitely because the squeezing due to chaotic instability and the spreading due to diffusion will lead to a standoff characterized by

$$
\sigma_{p}(0) \exp (-\lambda t) \simeq \sqrt{2 D t} ;
$$

this allows to estimate the time at which the fragmentation process is stopped, i.e.

$$
t_{C G}=\frac{1}{\lambda} \ln \left(\frac{\sigma_{p}(0) \sqrt{\lambda}}{\sqrt{2 D}}\right) .
$$

The condition necessary to make the quantum-classical correspondence valid is therefore

$$
t_{C G}<t_{Q} .
$$

If the noise intensity $D$ is large enough as to fulfill this condition, the Wigner quasiprobability never becomes so finely structured in momentum as to make the Moyal terms important. The initial classical state remains smooth and so the quantum coherence length remains small.

These arguments have been criticized by some authors [4, 16]. Casati and Chirikov [4] noted that the original arguments of Zurek and Paz are oversimplified since they refer to an autonomous one-dimensional system, which cannot be chaotic. They use an inverted parabola which is classically unstable but nonchaotic [5]. A more serious criticism of . was already predicted by Berman and Zaslavski [26] and Berry et al. [27] and that no significant departure of quantum from classical mechanics has been noticed to occur at this time: consequently no problem to the correspondence principle is created. Casati and Chirikov point out the benefits of adopting the Husimi rather than the Wigner quasi-probability distribution. The Husimi distribution is a Gaussian smoothing of the Wigner quasiprobability and the quantum fine structures are washed out by this coarse-graining procedure. As a consequence, even if at a logarithmic time, according to Zurek and Paz, the Wigner quasi-probability significantly departs from the corresponding classical probability distribution, the Husimi distribution is still very close to the classical prediction, and consequently the correspondence principle is safe. According to Casati and Chirikov, the departure of the classical from quantum predictions is expected to take place at the much longer relaxation time $t_{R}$, which is proportional to some power of $(A / \hbar)$ 沺.

It has to be pointed out, however, that the Husimi distribution is equivalent to the Wigner distribution, and that both are equivalent to ordinary quantum mechanics. Thus, the validity of the correspondence principle cannot depend only on the adoption of the former rather than the latter distribution. The reasons for the lack of any significant departure of quantum from classical predictions has been discussed in detail by Roncaglia et al. 28] and by Bonci et al. [29] and the conclusion was reached that this is essentially due to the fact that a Gibbs ensemble picture is adopted and the attention is focused only on expectation values of dynamical variables, which are coarse-grained quantities, typically not very sensitive to quantum coherence effects [32].

The work of Farini, Boccaletti and Arecchi [16] criticizes another aspect of the Zurek and Paz argument: classical chaos is characterized by stretching and folding and Ref. [5] neglects folding, i.e. neglects the possibil- 
ity of rapid twisting of the phase space direction corresponding to the local maximum Lyapunov exponent. In this case a given variable, say the momentum, does not experience only squeezing but both expansion and contraction, depending upon the local position on the trajectory. These authors supported their arguments by numerically studying a one-dimensional chaotic system, i.e. a double-well potential under the influence of a coherent perturbation. They found a parameter region in which the twisting process is important so that the nonclassical Moyal term does not become comparable to the classical term at the transition time $t_{Q}$, or, equivalently, no transition from classical to quantum mechanics occurs at the time predicted by [26] and [27] as well as by [5].

\section{MECHANICS AND THERMODYNAMICS: A GENERAL PERSPECTIVE}

In this Section we address the same problem as that of Section II from a different perspective, still heuristic, but more general than that adopted by Zurek and Paz. This will serve the useful purpose of bypassing, at least in part, the criticisms of Refs. [4, 16. In fact we shall show that the conclusions of Zurek and Paz are correct provided that the strong chaos condition is ensured.

We adopt the same picture as that used by Zaslavsky in 25 to illustrate the difference between purely ergodic motion and motion with mixing. We consider an $N$ dimensional classically chaotic system and a single trajectory moving within the phase space region $S(E)$ compatible with a given energy $E$. Let us denote by $M(t)$ the unexplored area, namely the area of the phase space region $S(E)$ not yet explored by this trajectory at a given time $t$. In the mixing case 25] it is expected that $M(t)$ tends to zero exponentially for $t$ tending to infinity.

We think that a more general picture can be obtained by using the arguments by Tsallis and co-workers [30,31. These authors are trying to establish a unified approach to nonlinear dynamics, extending from strong to weak chaos, based on the generalization of the property of exponential sensitivity to initial conditions. Their arguments rest on the connection between this generalized property and the entropic index $q$ as well as on numerical calculations which support their conjecture. By adapting their approach to the calculation of $M(t)$, we obtain [33].

$$
M(t)=\frac{M(0)}{\left[1+\lambda_{q} t(1-q)\right]^{\frac{1}{1-q}}} .
$$

The case of strong chaos corresponds to $q=1$, which makes the decay of $M(t)$ exponential, while the more general case of weak chaos corresponds to $q<1$ [33. If we now quantize this system, the quantum manifestations of the system can be neglected if the size of the wavepacket is small compared to $M(0)$. However, even in this case, it is not possible to disregard the quantum effects when
$M(t)$ becomes of the order of $(\hbar)^{N}$ : at this time quantum interference between two different portions of the classical trajectory becomes important. This condition defines the quantum-classical correspondence breakdown time $t_{Q}$ as:

$$
M\left(t_{Q}\right)=\hbar^{N}
$$

It is straightforward to show that:

$$
t_{Q}=\frac{1}{\lambda_{1}} \ln \left(\frac{M(0)}{\hbar^{N}}\right)
$$

if the strong chaos condition $q=1$ is realized, and

$$
t_{Q}=\frac{1}{\lambda_{q}(1-q)}\left[\left(\frac{M(0)}{\hbar^{N}}\right)^{1-q}-1\right]
$$

if the weak chaos condition $q<1$ is fulfilled. Therefore, we see from Eq. (9) that we recover the logarithmic dependence of [5,26,27] on $\hbar$ (in the more general $N$ - dimensional case) only in the case of strong chaos, $q=1$, while in a more generic condition of weak chaos, $q<1$, the dependence of $t_{Q}$ on $\hbar$ is given by an inverse power law, as shown by Eq. (10). In the case where the action $M(0)$ is macroscopic, this means a significant increase of the time of transition from classical to quantum mechanics.

In our opinion, the above results endorse the arguments by Zurek and Paz and lend them protection from the criticism of Farini et al. [16] if not completely from those of Casati and Chirikov 4 . In the one-dimensional case $N=1$, our prediction for $t_{Q}$ in the strong chaos case becomes practically identical to the Zurek and Paz result, Eq. (3). As to the criticism raised in [16], Eqs. (9) and (10) naturally suggest that the parameter region considered there, where the twisting process is important, actually might correspond to a situation of weak chaos in which the transition from classical to quantum physics is significantly postponed in time.

Our argument does not make the conclusions of Zurek and Paz on the physical significance of $t_{Q}$ safe from the main criticism of Ref. [4], concerning, as we have seen, the fact that no significant difference between quantum and classical predictions shows up at the logarithmic time. As explained in detail in [28,29, this criticism is essentially correct (see however [32]) if applied to the measurement of smooth variables or to the observation of other coarse-grained quantities. However, we cannot rule out the possibility that the breakdown of the correspondence principle, if no use of $D \neq 0$ is made, can be detected, in principle, by means of a different kind of observation. Thus, in spite of the criticism of Casati and Chirikov, we believe that this breakdown would be signalled by the substantial difference between the Wigner function and the corresponding classical probability distribution. We also note that this departure of the quantum from the classical distribution occurs at a relatively short time, 
if the strong chaos condition making $t_{Q}$ logarithmically dependent on the Planck constant is adopted.

We think in fact that the use of the Husimi distribution, suggested in [4], is misleading. It is well known that, because of its coarse-grained nature, the Husimi function (or $Q$ function in the quantum optics language) cannot be used as a convenient visual representation of the quantum coherence. It fails signalling quantum coherence even when this becomes macroscopic. The birth of quantum coherence, on the contrary, is clearly signalled by the wild oscillations of the Wigner function, and by its negative values as well. A clear example is provided by the linear superposition of two spatially separated coherent states: while the Wigner function associated to it is quite different from that associated to the corresponding statistical mixture, with the use of the Husimi function we would be practically unable to distinguish one case from the other.

More generally speaking, in our opinion, any formal mathematical procedure, inspired to the coarse-graining procedures, but not corresponding to a real physical process, independent of the observer, is unsatisfactory because it is ultimately justified only by the limitation of a given observation process, and the convolution used to process coarse graining can always be inverted so as to recover the original fine-grain picture. Furthermore, even setting aside the possibility of overcoming the limitations of a given observation process, we are made to feel uncomfortable by a theoretical perspective where the correspondence principle is made safe by a human dependent coarse-graining process rather than by a genuine property of nature. For these reasons we are forced to look for a theoretical perspective where the coarse graining process is made by nature, rather than by the observer.

The need for this choice is reinforced if we move to treat the second of the two problems under discussion in this letter: the derivation of the second law of thermodynamics from mechanics. As well known, the standard mechanical entropy, the Gibbs entropy associated to the Liouville density $\rho(\mathbf{X}, t)$ of the classical phase space

$$
S_{G}(t)=\int_{\Gamma} d \mathbf{X} \rho(\mathbf{X} ; t) \ln \rho(\mathbf{X} ; t),
$$

is a constant of motion because of the conservation of phase space volumes in Hamiltonian systems. Irreversibility and, correspondingly, an increasing entropy (i.e., the second law of thermodynamics) can be derived from a dynamical picture only by replacing the Liouville density by its coarse-grained version $p(\mathbf{X}, t)$, and this latter is obtained by averaging $\rho(\mathbf{X}, t)$ over a suitably small phase-space volume. In the case of a classically chaotic system this coarse-graining is a quite natural request for a human observer, since, if the mixing assumption is made [25], an initial condition given by a smooth Liouville density is changed into a highly fragmented distribution $\rho(\mathbf{X}, t)$. The coarse-graining process changes the Liouville density $\rho(\mathbf{X}, t)$ into the smooth $p(\mathbf{X}, t)$, and this is indistinguishable from the conventional microcanonical distribution: as a remarkable effect of all this, the
Gibbs entropy is naturally changed into the Boltzmann entropy.

In our opinion, deriving thermodynamics from classical mechanics by making recourse to observer's limitations is unsatisfactory. A much better perspective would be given by the coarse-graining process occurring as a genuinely phenomenon of nature rather than as a merely mathematical procedure reflecting observer's limitations. The theoretical proposal of Zurek and Paz is attractive because it affords an illuminating example of physical (rather than subjective and merely mathematical) coarse-graining process. In fact, the time $t_{C G}$ of Eq. (5), at which the fragmentation process induced by chaos is stopped by diffusion, is not only the time that must become shorter than $t_{Q}$ to prevent the breakdown of the quantum-classical correspondence. This is also the time at which the coarse-graining process, produced by nature, changes the incompressible Hamiltonian fluid into a fluid dynamic breaking the Liouville theorem, thereby making the entropy of the chaotic system increase [5, [7]. This is the reason why we have used the notation $t_{C G}$. This time signals the transition from mechanics to thermodynamics.

This coarse-graining time can be derived in a general way using again the above argument based on the unexplored area $M(t)$. In fact, in the presence of an isotropic diffusion process with diffusion coefficients $D_{x}$ and $D_{p}$ for the space and the momentum, respectively, the phase space is practically filled by the trajectory and a coarsegraining process is completed when the condition

$$
M\left(t_{C G}\right)=\left(2 \sqrt{D_{x} D_{p}} t_{C G}\right)^{N}
$$

is realized. Solving the equation for $t_{C G}$, we obtain

$$
t_{C G}=\frac{1}{\lambda_{1}} \ln \left(\frac{M(0) \lambda_{1}^{N}}{\left(2 \sqrt{D_{x} D_{p}}\right)^{N}}\right)
$$

if the case of strong chaos, and

$$
t_{C G}=\frac{1}{\lambda_{q}(1-q)}\left[\left(\frac{M(0) \lambda_{q}^{N}}{\left(2 \sqrt{D_{x} D_{p}}\right)^{N}}\right)^{1-q}-1\right]
$$

in the case of weak chaos. We see that, as in the case of $t_{Q}$, the adoption of the weak chaos condition makes $t_{C G}$ much larger (notice that $M(0)$ is a macroscopic quantity) and that its expression reduces to that of Eq. (5), the same as that derived by Zurek and Paz in the onedimensional case, if the assumption of strong chaos is made and only the momentum variable is assumed to undergo the influence of external fluctuations.

\section{SPONTANEOUS FLUCTUATIONS AS A SOURCE OF THE CLASSICALITY OF THE SOLAR SYSTEM DYNAMICS}

In the preceding Section we have seen that the introduction of fluctuations (of environmental origin accord- 
ing to the theoretical perspective adopted in Refs. [5]) is equivalent to establishing a coarse-graining process, and that this yields a natural derivation of the second law of thermodynamics from mechanics. In this section we prove that the same important effects are produced by fluctuations of different origin: these are those postulated by the so-called spontaneous reduction models [17 20]. These models are modifications of quantum mechanics with negligible effects at the microscopic level, where standard quantum theory still holds true. At the macroscopic level, on the contrary, these corrections cause a fast collapse of the linear superposition of distinct physical properties, so that, in accordance with our daily experience, any single state is associated to only one value of a given physical property: the paradoxical condition denoted as Schrödinger's cat is made impossible. The first model of this kind was proposed by Ghirardi, Rimini e Weber [17, who assumed that each particle is subject to abrupt and spontaneous localizations with mean rate $\lambda_{G R W} \simeq 10^{-16} \mathrm{sec}^{-1}$ and localization length $a \simeq 10^{-5} \mathrm{~cm}$. The rate $\lambda_{G R W}$ and the length $a$ are to be regarded as two new constants of nature. We shall refer to this as GRW model. This model was later improved by Ghirardi, Pearle and Rimini $[18$ to take into account that the particles are indistinguishable, and the instantaneous localization process was replaced by a continuous version described by a Wiener process with intensity $\gamma_{G P R} \simeq 10^{-30} \mathrm{~cm}^{-3} \mathrm{sec}^{-1}$. This model will be denoted by us as GPR model. At the same time, Diósi and independently Penrose, [19], tried to elaborate a reduction model based on gravity without introducing any new constant of nature; however Ghirardi, Grassi and Rimini [20] showed that these models resulted in an exceedingly large energy increase, and amended the model from this fault by introducing a mass smearing over volumes of the order of $a^{3} \simeq 10^{-15} \mathrm{~cm}^{3}$ ( $a$ is again the new constant that according to the GRW theory establishes the space scale within which the wave function reduction occurs), so as to keep the energy increase within experimental constraints. Therefore, this latter version of the model of Ref. [20] only requires one new constant of nature, the length $a$, is perfectly consistent with experimental constraints and can be physically explained as an effect of spontaneous gravitational potential fluctuations of dipole type [34]. We shall refer to this version of the theory of spontaneous fluctuations as GGR model.

The introduction of a new stochastic term in the Schrödinger equation is equivalent to adding a new, and fundamental, diffusion-like process to the dynamics of systems and for this reason the effects of spontaneous reduction models are analogous to those produced by the environment-induced decoherence. The main difference with standard thermal master equations (of environmental origin) is that no friction term is associated to these reduction models, and this is responsible for the steady energy increase, a property characterizing all these models. To illustrate formally the nature of these models, let us consider the quantum dynamics of the center of mass of a macroscopic body of mass $M$, volume $V$ and with a number $N$ of constituent particles. According to all the reduction models the dynamics of the center of mass is almost completely decoupled from the internal dynamics. Thus we can represent the dynamics of the center of mass coordinate $\vec{q}$ by means of an equation of motion for its reduced density matrix $\rho\left(\vec{q}, \vec{q}^{\prime}, t\right)$. This equation reads: ,

$$
\frac{\partial \rho\left(\vec{q}, \vec{q}^{\prime}, t\right)}{\partial t}=\frac{i}{\hbar}\left\langle\vec{q}\left|\left[\rho, H_{C M}\right]\right| \vec{q}^{\prime}\right\rangle-\Gamma\left(\left|\vec{q}-\vec{q}^{\prime}\right|^{2}\right) \rho\left(\vec{q}, \vec{q}^{\prime}, t\right),
$$

where $\Gamma$ is a function of $\left|\vec{q}-\vec{q}^{\prime}\right|^{2}$ dependent upon the chosen reduction model. The explicit form of $\Gamma$ is:

$$
\Gamma_{G R W}\left(\left|\vec{q}-\vec{q}^{\prime}\right|^{2}\right)=N \lambda_{G R W}\left(1-\exp \left[-\frac{\left|\vec{q}-\vec{q}^{\prime}\right|^{2}}{4 a^{2}}\right]\right)
$$

for the original GRW model 17,

$$
\Gamma_{G P R}\left(\left|\vec{q}-\vec{q}^{\prime}\right|^{2}\right)=\frac{\gamma_{G P R}}{2} \int d^{3} x\left[F(\vec{q}-\vec{x})-F\left(\vec{q}^{\prime}-\vec{x}^{\prime}\right)\right]^{2}
$$

for the GPR model of Ref. [18], where $F(\vec{q})$ is a function depending on the particle number density at the point $\vec{q}+\vec{y}, D(\vec{y})$ [18], defined by:

$$
F(\vec{q}-\vec{x})=\int d^{3} y \frac{D(\vec{y})}{(2 \pi)^{3 / 2} a^{3}} \exp \left[-\frac{|\vec{q}-\vec{x}+\vec{y}|^{2}}{2 a^{2}}\right] ;
$$

finally for the GGR model, namely the gravitationallyinduced reduction model of [19] amended from the excessive energy increase in 20], we have

$$
\Gamma_{G G R}\left(\left|\vec{q}-\vec{q}^{\prime}\right|^{2}\right)=-\frac{1}{\hbar}\left[U(0)-U\left(\left|\vec{q}-\vec{q}^{\prime}\right|\right)\right] ;
$$

where $U\left(\left|\vec{q}-\vec{q}^{\prime}\right|\right)$ is the gravitational interaction energy between two copies of our macroscopic body with the center of mass placed at $\vec{q}$ and $\vec{q}^{\prime}$. The evolution equation Eq. (15) clearly shows that the new term induces a rapid decay of the quantum coherence between wave-functions components separated by distances larger than the new length constant $a$.

These correction terms are nothing but diffusion-like terms and this can be clearly seen if one rewrites Eq. (15) for $\rho\left(\vec{q}, \vec{q}^{\prime}, t\right)$, in terms of the Wigner function $W(x, p)$. In fact multiplication by $\left|\vec{q}-\vec{q}^{\prime}\right|^{2}$ in the coordinate representation is equivalent to the application of the Laplacian operator in momentum space in the Wigner representation [24],

$$
\left|\vec{q}-\vec{q}^{\prime}\right|^{2} \rho\left(\vec{q}, \vec{q}^{\prime}\right) \leftrightarrow-\nabla_{p}^{2} W(\vec{x}, \vec{p}) ;
$$

and therefore these spontaneous reduction models are equivalent to adding the diffusion-like operator 


$$
-\Gamma\left(-\nabla_{p}^{2}\right) W(\vec{x}, \vec{p})
$$

to the evolution equation of the Wigner function $(\Gamma$ can always be expressed as a power series and $\Gamma(0)=0$ ). Since the effects of environmental decoherence are established by Zurek and Paz in the reversible classical limit, where dissipation is negligible compared to diffusion, we can apply to the new cases of this Section the same arguments as those illustrated in Section II, with no problem whatsoever. This is equivalent to stating that also the spontaneous fluctuations allow us to protect the classical-like system from an early transition to the quantum regime, induced by deterministic classical chaos. It is possible to realize the key condition $t_{C G}<t_{Q}$, which makes it impossible for the transition from classical to quantum mechanics to occur at any time, so as to make the quantum-classical correspondence safe. In fact for both the spontaneous reduction models and the environment-induced decoherence we derive the following evolution equation for the dispersion $\Delta p_{i}(t)$ of a given momentum component of the macroscopic body ,

$$
\frac{d}{d t}\left(\Delta p_{i}(t)\right)^{2}=\langle\ldots\rangle_{Q}+2 D,
$$

where $\langle\ldots\rangle_{Q}$ stands for the quantum Hamiltonian terms and the diffusion coefficient $D$ depends on the model chosen according to the prescriptions of Table $\mathbb{1}$.

TABLE I. Expression of the diffusion coefficients for the various models. The subscripts env, GRW, GPR and GGR denote the fluctuation intensities predicted by refs. [0, 17, 18, 20, respectively. $G$ is the gravitational constant and $S_{\perp}$ denotes the surface of the body, transverse to the direction of $p_{i}$.

\begin{tabular}{|l|c|c|c}
\hline \hline$D_{\text {env }}$ & $D_{G R W}$ & $D_{G P R}$ & $D_{G G R}$ \\
\hline $2 M \gamma k T$ & $\frac{N \lambda_{G R W} \hbar^{2}}{4 a^{2}}$ & $\frac{\gamma_{G P R} \hbar^{2} N^{2} S_{\perp}}{4 V^{2} a \sqrt{\pi}}$ & $\frac{G \hbar M^{2}}{2 V}$ \\
\hline \hline
\end{tabular}

The resulting linear increase in time of the momentum dispersion due to diffusion is the key ingredient for the determination of $t_{C G}$. Using the same arguments as those of Sections II and III we derive for $t_{C G}$ the same expression as that of Eq. (5), with the same dependence on $D$. The prescriptions to adopt to evaluate $D$ is dictated by the model adopted, as indicated in Table 1 .

Let us now consider again the classically chaotic celestial systems described in the introduction and discussed in [7], and let us focus on the solar system. According to the discussion of the preceding Section, if the motion of the solar system as a whole satisfies the strong chaos condition (with the global Lyapunov exponent evaluated in 114.15), the logarithmic time prediction derived by Zurek in 7] is correct and the present quantum state of the solar system would be characterized by planetary wave functions spreading all over their orbits. However, as shown above, even a tiny diffusion process is sufficient to recover classicality, namely a time $t_{C G}$ shorter than the breakdown time $t_{Q}$. When making the estimates discussed in [7], Zurek considers for simplicity only the motion of the most massive planet, Jupiter, and considers the environmental diffusion due to the interaction of Jupiter with the interplanetary gas in its vicinity (of approximate number density $0.1 \mathrm{~cm}^{-3}$ ). This yields $\gamma \simeq 10^{-26} \mathrm{sec}^{-1}$. Then, using Jupiter surface temperature $T \simeq 100^{\circ} \mathrm{K}$, we get

$$
D_{\text {env }} \simeq 10^{-10} \mathrm{erg} \cdot \mathrm{gr} / \mathrm{sec} .
$$

By identifying the initial momentum dispersion $\sigma_{p}(0)$ with Jupiter's mean momentum, and so overestimating $t_{C G}$, we get from Eq. (5) $t_{C G} \simeq 3 \cdot 10^{8}$ years $<t_{Q} \simeq 7 \cdot 10^{8}$ years. Although the coarse-graining time is extremely long, the transition to quantum mechanics is made impossible by the condition $t_{C G}<t_{Q}$ : initial classical dynamics of the solar system will always remain classical. Now we can apply the same estimate procedure to the diffusion induced by spontaneous fluctuations, using the expressions for the corresponding diffusion coefficients given by Table 1 . Using again Jupiter values for $M, V$ and $N$, and the spontaneous reduction parameter values, we obtain the values of Table II.

TABLE II. Numerical estimates for the diffusion coefficients for the environmental decoherence and for the spontaneous reduction models expressed in erg.gr/sec. The mean-

\begin{tabular}{|c|c|c|c|}
\hline$D_{e n v}$ & $D_{G R W}$ & $D_{G P R}$ & $D_{G G R}$ \\
\hline $10^{-10}$ & $10^{-8}$ & $10^{-11}$ & $10^{-4}$ \\
\hline
\end{tabular}
ing of the subscripts is the same as that of the corresponding subscripts in Table 
With the joint use of the results reported in Table I and of the same argument as that applied to the case of environmental fluctuations, all these models are proved to share with the theory of environmental fluctuations the attractive property of yielding $t_{C G}<t_{Q}$, making it impossible for the solar system to fall in a quantum condition. This is a natural consequence of the fact that, whatever the origin of fluctuation is, either spontaneous or environmental, the dynamics of the system is described by Eq. (11), with the friction term neglected. The most interesting and surprising fact is that this celestial dynamics example is the first realistic situation in which spontaneous reduction effects (especially those of the gravitational model) are found to be much stronger than those associated to environment-induced decoherence. In fact a number of estimates of spontaneous reduction effects exist in literature [11,21 23, 35] and all of them agree on the fact that these effects are blurred by environmental decoherence. This fact has led to the common criticism that these models are a useless modification of quantum mechanics. This would be so because these theories are made to pass all the experimental tests by an ad hoc choice of parameters, which, at the same time, makes them practically unverifiable [21], since their effect is always blurred by decoherence.

However, we have to point out that the results concerning the solar system here discussed are not a compelling evidence of the gravitationally-induced objective reduction model, since no experiment can be made to assess if the solar system dynamics are made classical by the environment-induced decoherence or by the spontaneous gravitational fluctuations. Is there, in this condition, any deep reason to trace back the fluctuations necessary to maintain the classicality of macroscopic systems to the corrections to ordinary physics rather than to the environmental influence? The answer to this important question is in positive. Let us see why. First of all, as explained in [10,11] the theory of environmental decoherence provides only a statistical solution to the problem of the emergence of classicality, in the sense that it only shows the emergence of statistical mixtures of classically distinguishable events from quantum dynamics. This theory does not explain the objectification problem [36], the uniqueness of events, i.e. how a single localized event out of the many which are statistically possible is realized. On the contrary, this emergence of individual events is well explained by spontaneous reduction models whose fundamental equation is a stochastic modification of the Schrödinger equation: the emergence of an event corresponds to a single stochastic process in the Hilbert space of the Universe. Another argument making objective reduction theories preferable, in our opinion, to the approaches based on environmental decoherence is connected to the problem described in section III, i.e. the derivation of the second law of thermodynamics from mechanics, as an effect of the coarse-graining on the classical phase space. As we have already said, a process of coarse graining realized by nature is more satisfactory than an equivalent mathematical procedure forced by the observation limitations. The theory of environment decoherence is not totally free from this subjective aspect. First of all, we note that the environmental fluctuation is derived from within the ordinary quantum mechanics by making a contraction on the environmental degrees of freedom: a subtle way of stating the limitations affecting human observation. Moreover the diffusion and the relaxation terms are derived assuming the environment to be in a state of thermodynamical equilibrium. This means that an implicit thermodynamical assumption is made, even if this serves only the purpose of realizing a coarsegraining condition. We cannot conclude that thermodynamics is genuinely derived from dynamics, since the known procedures used to derive fluctuation-dissipation processes rest already on thermodynamics. It is better to make a frank admission that the assumptions made correspond to correcting ordinary physics, and that the diffusion process necessary to realize the "objective coarsegraining" are something new, a stochastic seed foreign to ordinary dynamics. This frank admission is made explicit by the theories of spontaneous fluctuations.

\section{CONCLUSIONS}

The main results of this letter are the following:

1. We have presented a general, even though heuristic, argument, confirming the logarithmic dependence on $\hbar$ of the time of transition from classical to quantum mechanics, established by the authors of Refs. [5]. The derivation of this letter shows that the condition of strong chaos is a fundamental requirement for this prediction to be correct, and make the original conclusions of Zurek and Paz more robust against the criticism of [4, 16.

2. We have shown that the gravitationally-induced objective reduction model of $[19,20]$, applied to the chaotic dynamics of the solar system, yields a transition from quantum to the classical domain, which is much faster than that produced by the environmental fluctuations.

This fact is not a compelling evidence of the existence of spontaneous fluctuations. In fact, the expression of the various diffusion coefficients (see Table I) shows that one needs very massive objects with an extremely weak dissipation (like planets) to make the objective reduction effects prevail on environmental decoherence: a condition certainly exceeding those of the experimental observation in an ordinary laboratory. Thus, it seems impossible at the moment, to assess experimentally whether the classical motion of macroscopic systems close to the conditions of the solar system, is due to environmental or to spontaneous decoherence. We note furthermore that the condition of weak chaos has the effect of postponing 
both the transition from classical to quantum mechanics and the coarse graining time $t_{C G}$. However, in this condition the transition time turns out to be much more sensitive to the intensity of the fluctuations. This means that, in principle, it would be much easier to distinguish the environment-induced decoherence process, whose intensity is expected to be temperature dependent, from the influence of spontaneous collapses, whose intensity is temperature independent. In other words, weak chaos might offer the proper conditions to assess experimentally the existence of spontaneous fluctuations.

[1] D. Giulini, E. Joos, C. Kiefer, J. Kupsch, I.O. Stamatescu, M.D. Zeh, Decoherence and the appearance of classical world in quantum theory, (Springer, Berlin 1996).

[2] E. Schrödinger, Naturwissenschaften 23, 807, 823, 844 (1935).

[3] Quantum Theory and Measurement, J.A. Wheeler and W.H. Zurek eds., (Princeton University Press, Princeton, NJ, 1983).

[4] G. Casati and B.V. Chirikov, Phys. Rev. Lett. 75, 350 (1995).

[5] W.H. Zurek, J.P. Paz, Phys. Rev. Lett. 72, 2508 (1994).

[6] W.H. Zurek and J.P. Paz, Physica D83, 300 (1995).

[7] W.H. Zurek, Proceedings of Nobel Symposium 104, Physica Scripta, in press (e-print quant-ph/9802054).

[8] P. Grigolini, Quantum Irreversibility and Measurement, World Scientific, Singapore (1993).

[9] W.H. Zurek, Phys. Rev. D 24, 1516 (1981); ibid. 26, 1862 (1982); E. Joos, and H.D. Zeh, Z. Phys. B 59, 229 (1985).

[10] See the debate raised by W.H. Zurek, Phys. Today 44 (10), 36 (1991) and reported in Phys. Today 46 (4), 13 (1993); R. Omnés, Phys. Lett. A 187, 26 (1994); R. Omnés, in: New Developments on Foundation Problems in Quantum Physics, M. Ferrero, A. van der Merwe eds. (Kluwer, Dordrecht 1997) pag. 275.

[11] L. Bonci, P. Grigolini, G. Morabito, L. Tessieri, D, Vitali, Phys. Lett. A 209, 129 (1995).

[12] J. Wisdom, S.J. Peale and F. Maignard, Icarus 58, 137 (1984).

[13] J. Wisdom, Icarus, 63, 272 (1985).

[14] J. Laskar, Nature 338, 237 (1989).

[15] G.J. Sussman and J. Wisdom, Science 257 (1992).

[16] A. Farini, S. Boccaletti, and F.T. Arecchi, Phys. Rev. E 534447 (1996).

[17] G.C. Ghirardi, A. Rimini, T. Weber, Phys. Rev. D 34, 470 (1986).

[18] G.C. Ghirardi, P. Pearle, A. Rimini, Phys. Rev. A 42, 78 (1990).

[19] L. Diósi, Phys. Rev. A 40, 1165 (1989); R. Penrose, Gen. Rel. Grav. 28, 581 (1996).

[20] G.C. Ghirardi, R. Grassi, A. Rimini, Phys. Rev. A 42, 1057 (1990).
[21] M. Tegmark, Found. Phys. Lett. 6, 571 (1993).

[22] L. Tessieri, D. Vitali, P. Grigolini, Phys. Rev. A 51, 4404 (1995).

[23] S. Bose, K. Jacob and P.L. Knight, e-print quant$\mathrm{ph} / 9712017$.

[24] A.O. Caldeira and A.J. Leggett, Physica 121A, 587 (1983); F. Haake and R. Reibold, Phys. Rev. A 32, 2462 (1985); W.G. Unruh and W.H. Zurek, Phys. Rev. D 40, 1071 (1989); B.L. Hu, J.P. Paz and Y. Zhang, Phys. Rev. D 45, 2843 (1992).

[25] G.M. Zaslavsky, Chaos in Dynamic Systems (Harwood Academic Publishers, Longhorne, 1985).

[26] G.P. Berman and G.M. Zaslavsky, Physica A 91, 450 (1978).

[27] M.V. Berry, N.L. Balasz, M. Tabor, and A. Voros, Ann. Phys. 122, 26 (1979).

[28] R. Roncaglia, L. Bonci, B.J. West and P. Grigolini, Phys. Rev. E 51, 5524 (1995).

[29] L. Bonci, P. Grigolini, A. Laux and R. Roncaglia, Phys. Rev. A 54, 112 (1996).

[30] C. Tsallis, A.R. Plastino and W.-M. Zheng, Chaos, Solitons and Fractals, 8885 (1997).

[31] M.L. Lyra and C. Tsallis, Phys. Rev. Lett. 80, 53 (1998).

[32] In a very recent paper by S. Habib, K. Shizume and W.H. Zurek, Phys. Rev. Lett. 80, 4361 (1998), a deviation between classical and quantum expectation values is found at times compatible with the logarithmic timescale, but the insufficient parameter range considered does not allow definitive conclusions.

[33] We make the plausible assumption that $M(t)$ is inversely proportional to the increase of the distance between two distinct trajectories starting from very close initial conditions. Tsallis and co-workers 30,31 study also the dynamics corresponding to attractive fixed points, with $q>1$. For the main purpose of this letter only the case $q<1$ is of interest.

[34] P. Pearle and E. Squires, e-print quant-ph/9503019.

[35] A. Rimini, in: Advances in Quantum Phenomena, NATO ASI Series B vol. 347, E.G. Beltrametti and J.M.LevyLeblond eds., pag.321.

[36] P. Busch, P.J. Lahti and P. Mittelstaedt, Quantum theory of measurement, (Springer, Berlin 1991), pag. 6 . 